Original Paper http://ajol.info/index.php/ijbcs http://indexmedicus.afro.who.int

\title{
Performances de reproduction du poisson chat endogène du Cameroun Clarias jaensis (Boulenger, 1909) en milieu contrôlé
}

\author{
Paul ZANGO ${ }^{1 *}$, Minette Tabi Eyango TOMEDI ${ }^{1}$, Thomas Ewoukem EFOLE ${ }^{2}$, \\ Claudine Tekounegning TIOGUE ${ }^{3}$, David NGUENGA ${ }^{4}$, Stephane Michel KAMANKE \\ KAMANKE $^{1}$, Olivier MIKOLASEK ${ }^{5}$ et Joseph TCHOUMBOUE ${ }^{3}$ \\ 1 Université de Douala, Institut des Sciences Halieutiques, \\ Département d'Aquaculture, BP 7236, Bassa Douala, Cameroun. \\ ${ }^{2}$ Université de Dschang, Faculté d'Agronomie et des Sciences Agricoles (FASA), \\ Département de Foresterie, BP 222 Dschang, Cameroun. \\ ${ }^{3}$ Université de Dschang, Faculté d'Agronomie et des Sciences Agricoles (FASA), \\ Département des Productions Animales, BP 222 Dschang, Cameroun. \\ ${ }^{4}$ Institut de Recherche Agricole pour le Développement, \\ Unité de Recherche Piscicole, BP 255 Foumban, Cameroun. \\ ${ }^{5}$ UMR ISEM, CIRAD 34398 Montpellier cedex 5, France. \\ *Auteur correspondant, E-mail: paul1zango@hotmail.com;Tél.: (+237) 75389007
}

\section{RESUME}

Les performances de reproduction de Clarias jaensis ont été évaluées entre mai et octobre 2014 à Batié (Ouest-Cameroun). A cet effet, 30 femelles de poids moyen 199, $52 \pm 34,85 \mathrm{~g}$ ont été réparties en 5 lots comparables. Chaque lot choisi au hasard a été soumis à l'Hormone Chorionique Gonadotrophine (HCG) aux doses de 3500, 4000 et 4500 Unités Internationales (UI) / $\mathrm{kg}$ et aux extraits hypophysaires des mâles et des femelles. Le poids et le diamètre des ovocytes, les taux de fécondation et d'éclosion ont été évalués. Les principaux résultats ont montré que: le poids des ovocytes a été le plus élevé $(\mathrm{p}<0,05)$ avec l'extrait hypophysaire femelle $(39,33 \pm 17,08 \mathrm{~g})$; le diamètre des ovocytes a été comparable entre les traitements. Le taux de fécondation a été le plus élevé pour la dose de 4000 UI / kg de HCG (87, 50 $12,45 \%)$ suivi de l'extrait hypophysaire femelle $(85,08 \pm 14,29 \%)$. Cependant aucune différence significative $(\mathrm{p}>0,05)$ n'a été obtenue. Le taux d'éclosion a été plus élevé avec l'extrait hypophysaire femelle $(19,38 \pm 6,58 \%)$. La reproduction artificielle chez Clarias jaensis est possible avec l'hormone HCG à 4000 UI / kg ou des extraits hypophysaires femelles.

(c) 2016 International Formulae Group. All rights reserved.

Mots clés: Clarias jaensis, espèce endogène, performances de reproduction, captivité, Cameroun.

\section{Reproductive performances of indigenous catfish of Cameroon Clarias jaensis (Boulenger, 1909) in captivity}




\section{ABSTRACT}

In order to improve local biodiversity and number of fishes in aquaculture in Cameroon, reproductive performances in captivity of Clarias jaensis have been studied between Mai and October 2014 in Batié (West Cameroon). In fact, 30 females with $199,52 \pm 34,85 \mathrm{~g}$ were divided randomly into five comparable treatments. Each was submitted to a Human Chorionic Gonatropin Hormon at 3500, 4000 and 4500 Internationals Units (UI) / kg of body weight, males and female's pituitary extracts. The main results obtained were: The oocyte weight was significantly higher $(\mathrm{p}<0,05)$ with females' pituitary extracts $(39,33 \pm 17,08 \mathrm{~g})$, the oocyte diameter mean was comparable within the treatments. The higher fecundity rate was obtained with the Human Chorionic Gonadotropin Hormon at $4000 \mathrm{UI} / \mathrm{kg}$ of body weight (85, $08 \pm 14,29 \%)$. However, no significant differences $(\mathrm{p}>0,05)$ were found. The hatching rate was significantly higher $(\mathrm{p}<0,05)$ with the pituitary extracts of females $(19,38 \pm 6,58 \%)$. Clarias jaensis can be reproduced artificially using Human Chorionic Gonadotropin Hormon at 4000 UI / kg of body weight or female's pituitary extracts.

(C) 2016 International Formulae Group. All rights reserved.

Keywords: Clarias jaensis, reproductive performances, indigenous species, captivity, Cameroon.

\section{INTRODUCTION}

L'aquaculture, un des secteurs de la production alimentaire dont la croissance est la plus rapide doit jouer un rôle déterminant dans la satisfaction de la demande croissante de produits halieutiques (FAO, 2012). Pratiquée de manière responsable, elle offre des avantages durables en matière de sécurité alimentaire et de croissance économique à l'échelle mondiale (FAO, 2014). Cependant, Pour la plupart des espèces aquatiques, la pratique courante est basée sur la « chasse » aux géniteurs sauvages ou à la récolte des semences avec pour conséquences les risques d'introduction de maladies, de variabilité interannuelle de la production et la présence d'espèces parasites ou commensales qui peuvent se révéler être des pestes dans les conditions d'élevage (Sorgeloos, 2014). A cet effet, le besoin de diversification des produits piscicoles a conduit à la domestication des nouvelles espèces de poissons pour une meilleure valorisation et préservation de la biodiversité locale (Fostier et jalabert, 2004). C'est ainsi que de nos jours, près de 32 espèces endogènes de poissons sont élevées en pisciculture dans le Mékong (Lazard et al., 2009). Par contre, au Cameroun, à notre connaissance, il n'existe aucune espèce endogène véritablement domestiquée. L'utilisation $\mathrm{du}$ silure endogène Clarias jaensis en pisciculture constituerait non seulement un début de solution à l'insuffisance d'alevins de poissons chats mais aussi, contribuerait à la réduction de la pression de la pêche sur les espèces autochtones et à la préservation de la biodiversité endogène. Ainsi la maîtrise de la reproduction artificielle chez Clarias jaensis s'est révélé d'intérêt afin d'améliorer la productivité des systèmes de polyculture.

\section{MATERIEL ET METHODES}

\section{Zone d'étude}

L'étude s'est déroulée de mai à octobre 2014 à Batié (LN : 5 $17^{\circ} 0^{\prime}$ '- 5 $5^{\circ} 18^{\prime} 53^{\prime}$ ' et LE : $10^{\circ} 17^{\prime} 0^{\prime \prime}$ - $10^{\circ} 19^{\prime} 31^{\prime \prime}$ ') zone soudano guinéenne d'altitude de l'Ouest Cameroun.

\section{Origine et maintenance des géniteurs}

Un total de 131 géniteurs de Clarias jaensis $(255,70 \pm 105,60 \mathrm{~g}$ et $24,50 \pm 8,50$ $\mathrm{cm})$ pêchés à l'aide des nasses dans la rivière Ménoua à Santchou (LN $5^{\circ} 10^{\prime}-5^{\circ} 30^{\prime}, \mathrm{LE}$ $9^{\circ} 50^{\prime}-10^{\circ} 10^{\prime}$; altitude $700 \mathrm{~m}$ ) ont été 
utilisés. Ces géniteurs ont été transportés dans des alevinières à Batié et acclimatés pendant un mois dans un étang de $150 \mathrm{~m}^{2}$ intégré à une porcherie de $80 \mathrm{~m}^{2}$ sur pilotis contenant 10 porcs âgés de 5 mois.

\section{Sélection des géniteurs et induction hormonale}

Après acclimatation des géniteurs, 30 femelles et 30 mâles de poids et tailles moyens respectifs de 199,52 $\pm 34,85 \mathrm{~g}, 224 \pm$ $23,69 \mathrm{~g}, 28,33 \pm 2,90 \mathrm{~cm}$ et $33 \pm 2,02 \mathrm{~cm}$ ont été sélectionnés (Barras et Jobling, 2002). Les femelles ainsi sélectionnées ont été réparties au hasard en 5 lots comparables (poids et tailles) composés de 6 individus chacun. Trois des lots ont été traité à l'hormone hCG à raison de 3500, 4000 et $4500 \mathrm{UI} / \mathrm{kg}$ de poids vif pour chaque et deux à l'extrait hypophysaire (mâle et femelle). La préparation et l'administration des hormones ont été faites suivant les méthodes proposées par de nombreux auteurs (Barras et Jobling, 2002; Rukera, 2004 et Nguenga, 2000). Aussi bien pour l'hormone hCG que pour l'extrait hypophysaire, les femelles ont reçu deux injections intramusculaires respectives au $1 / 3$ et $2 / 3$ de l'hormone à injecter à 6 heures d'intervalle. Les femelles ainsi traitées ont été gardées individuellement dans des bidons plastiques perforés de 20 litres, numérotés et immergés dans un bac en béton. Les femelles ainsi conditionnées n'ont pas reçu d'aliments pendant $36 \mathrm{~h}$ à température de l'eau de $22^{\circ} \mathrm{C}$.

\section{Récolte des gamètes}

Après la maturation ovocytaire, les ovules de chaque femelle ont été récoltés par pression abdominale du haut vers le bas ou stripping (De Graaf et Janssen, 1996) dans les bols préalablement tarés et après récolte, chaque bol et son contenu (ovules) ont été pesés à l'aide d'une balance électronique de marque Kitchen scale SF-400 de sensibilité 0,1 g. Pour l'obtention de la laitance destinée à la fertilisation des ovules, les testicules ont été prélevés entièrement après sacrifice de chaque mâle répondant au critère de poids précédemment cité (sexe ratio $1: 1$ ), suivant la technique proposée par Cacot (2006).

\section{Conduite de l'essai}

La laitance obtenue des testicules a été prélevée à l'aide d'une seringue et mélangée aux ovules et ensuite une solution de fécondation ( $\mathrm{NaCl} 2 \mathrm{~g} / 1$ et urée $4 \mathrm{~g} / 1$ d'eau) a été utilisée pour rincer le mélange pendant 60 secondes. Ce mélange a été étalé sur les claies étiquetées préalablement aménagées dans les bacs d'incubation (hauteur d'eau = $0,37 \mathrm{~m}$ et le débit d'eau $=5,18 \mathrm{ml} / \mathrm{s}$ ). Un échantillon de 100 œufs a été prélevé dans chaque bol et disposé dans le bac d'incubation au même moment que les claies, ainsi qu'un autre bol contenant 100 ovules non fécondés préalablement prélevés avant le mélange à la laitance afin d'évaluer les taux de fécondation et d'éclosion de chaque femelle. Le bac d'incubation a été recouvert d'une bâche noire afin d'éviter le refroidissement nocturne. La température a été de $21 \pm 0,5{ }^{\circ} \mathrm{C}$ pendant l'incubation. Les larves après éclosion ont été maintenues dans le bac d'incubation, aucun apport d'aliment n'a été fait durant 4 jours (résorption vitelline chez Clarias jaensis). Les paramètres physico-chimiques de l'eau dans les bassines d'élevage (température, $\mathrm{pH}$, conductivité de l'eau,) ont été relevées hebdomadairement le matin, à midi et le soir à l'aide d'un multi-paramètre de marque Tech.

\section{Paramètres étudiés}

Le temps de latence a été calculé par différence entre l'heure d'injection 
hormonale et celle de la collecte des ovocytes (stripping), la durée d'incubation a été obtenue par intervalle de temps entre le début d'incubation et la fin de l'éclosion.

Diamètre ovocytaire $(\mathrm{mm})=$ moyenne de la distance sur 10 ovocytes mesurés sur papier millimétré) ;

Poids des ovocytes $(\mathrm{mg})$ a été obtenu par comptage du nombre d'ovocytes contenu dans un gramme de ponte ;

La fécondité absolue ( $\mathrm{Fa})$ a été calculée en multipliant le nombre total d'ovocytes dans $1 \mathrm{~g}$ par le poids total (g) de la ponte (Philippart, 1975 ; Dadébo et al., 2003).

La fécondité relative (Fr) a été évaluée en divisant le nombre total d'ovocytes pour un individu par son poids corporel (Pt) (Philippart, 1975).

Taux de fécondation $=($ Nombre d'œufs fécondés / Nombre total d'œufs incubés)*100

Taux d'éclosion $=$ (Nombre de larves obtenues / Nombre total d'œufs incubés)*100

\section{Analyse statistique}

Les résultats obtenus ont été soumis à l'analyse de la variance à un facteur (ANOVA1) et au test-t de Student. Le test de Duncan a permis de séparer les moyennes au seuil de 5\% lorsqu'il existait une différence significative. Le logiciel SPSS version 20 et le classeur EXCEL ont été utilisés.

\section{RESULTATS}

Diamètre et poids des ovocytes à différents types et doses d'hormones

Le diamètre des ovocytes a été plus élevé chez individus ayant reçu l'hormone HCG à 4500 UI/kg et les extraits hypophysaires males, la valeur la plus faible étant enregistrée chez ceux traités aux extraits hypophysaires femelles (Figure 1), cependant aucune différence significative $(p>0,05)$ n'a été observée entre les traitements. Le poids des ovules a été significativement plus élevé $(\mathrm{p}<0,05)$ avec l'extrait hypophysaire provenant des femelles comparé aux doses de 3500, 4000, 4500 UI/kg de 1'hormone HCG et à l'extrait hypophysaire mâle (Figure 2).

\section{Temps de latence et d'incubation}

Le temps de latence et la durée d'incubation ont diminué avec la température de l'eau (Tableau 1), les valeurs moyennes du temps de latence et de la durée d'incubation les plus élevées ont été respectivement de 31 et $59 \mathrm{~h}$ à $20,50 \pm 0,70{ }^{\circ} \mathrm{C}$ et les plus faibles de 13 et 22,5 h à $28,20 \pm 0,15^{\circ} \mathrm{C}$.

\section{Fécondités absolue et relative à différents types et doses d'hormone}

Les moyennes des fécondités absolue et relative sont illustrées par la Figure 3. Il en ressort que la moyenne de la fécondité absolue (Figure $3 \mathrm{~A}$ ) a été significativement plus élevée $(\mathrm{p}<0,05)$ avec l'extrait hypophysaire provenant de la femelle, suivie de l'hormone HCG. Elle a été comparable et faible entre l'extrait hypophysaire provenant du mâle et de HCG aux doses 3500 et 4500 UI / g de poids vif. Les valeurs moyennes de la fécondité relative (Figure $3 \mathrm{~B}$ ) ont été comparables entre l'extrait hypophysaire provenant de la femelle et de l'hormone HCG à la dose 4000 UI /g de poids vif et significativement élevée ( $\mathrm{p}<0,05)$ par rapport aux doses 3500, 4500 UI /g de poids vif et l'extrait hypophysaire du mâle.

\section{Taux de fécondation et d'éclosion à différents types et doses d'hormone}

Le taux de fécondation (Figure 4) le plus élevé a été obtenu avec la dose de 4000 UI/kg de l'hormone HCG suivi de l'extrait hypophysaire provenant des femelles. La 
valeur la plus faible étant obtenue avec l'extrait hypophysaire d'origine mâle. Cependant, aucune différence significative ( $p$ $>0,05)$ n'a été observée quel que soit le type et la dose d'hormones utilisées. Le taux d'éclosion a été significativement plus élevé $(\mathrm{p}<0,05)$ chez les individus ayant été traité avec les extraits hypophysaires femelles. Les valeurs les plus faibles ont été obtenues avec les doses de 4500 et $3500 \quad \mathrm{UI} / \mathrm{kg}$ respectivement.

Tableau1: Variation du temps de latence et d'incubation en fonction de la température de l'eau.

\begin{tabular}{lcc}
\hline Température de l'eau $\left({ }^{\circ} \mathbf{C}\right)$ & Latence $(\mathbf{h})$ & Incubation $(\mathbf{h})$ \\
\hline $20,50 \pm 0,70$ & $31,06 \pm 1,03$ & $59,00 \pm 0,11$ \\
$21,75 \pm 0,35$ & $30,53 \pm 0,51$ & $55,00 \pm 0,51$ \\
$22,50 \pm 0,70$ & $25,01 \pm 0,53$ & $47,75 \pm 0,26$ \\
$23,67 \pm 1,53$ & $24,00 \pm 0,13$ & $47,08 \pm 1,02$ \\
$24,00 \pm 0,00$ & $23,50 \pm 0,32$ & $28,80 \pm 1,10$ \\
$25,01 \pm 1,41$ & $22,58 \pm 0,15$ & $25,75 \pm 0,96$ \\
$27,00 \pm 0,91$ & $21,20 \pm 0,45$ & $24,00 \pm 0,51$ \\
$28,20 \pm 0,15$ & $13,00 \pm 0,12$ & $22,67 \pm 0,52$ \\
\hline
\end{tabular}

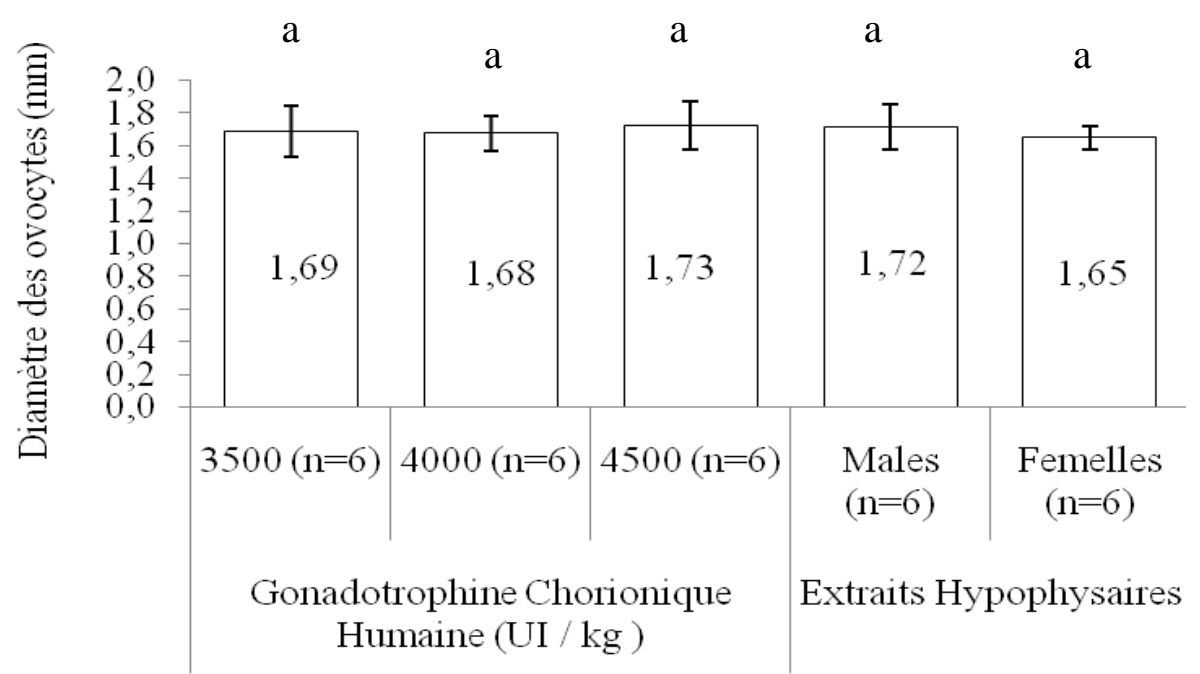

Types et doses d'hormones

Figure 1: Variation du diamètre des ovocytes en fonction du type et doses d'hormones. a: les histogrammes affectés de la même lettre ne sont pas significativement différentes $(\mathrm{p}>0,05) ; \mathrm{n}=$ nombre d'observations; $\mathrm{I}=$ écart type. 


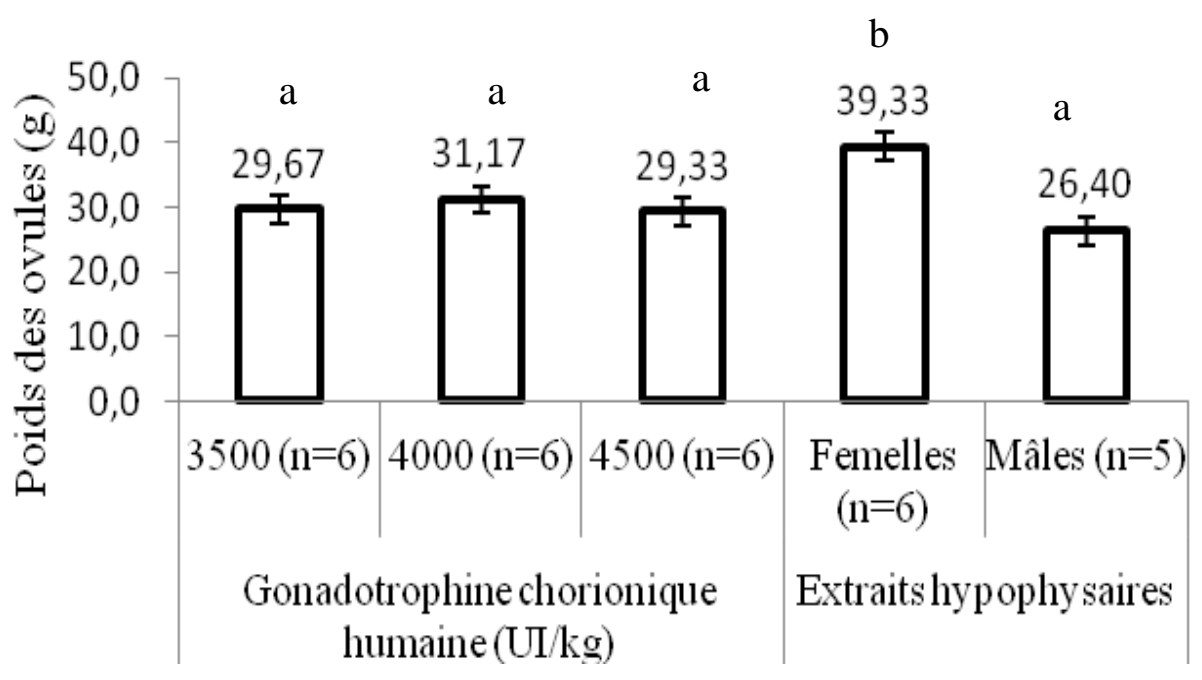

Figure 2 : Poids des ovocytes en fonction du type et dose d'hormones. a, b: les histogrammes affectés des mêmes lettres ne sont pas significativement différentes $(p>0,05) ; n=$ nombre d'observations; $I=$ écart type.

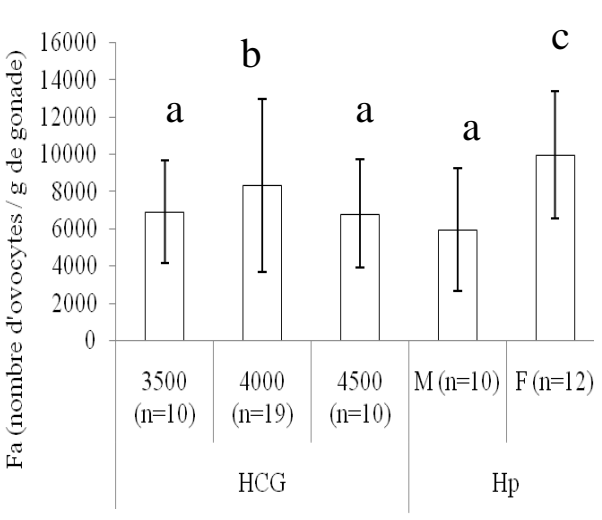

A

Types et doses d'hormones

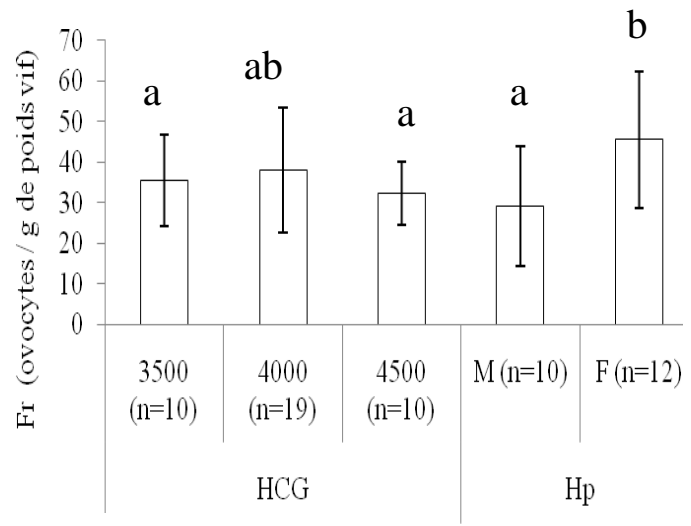

B Types et doses d'hormones

Figure 3: Variation de la Fécondité absolue (A) et de la Fécondité relative (B) en fonction du type et de la dose d'hormone. 


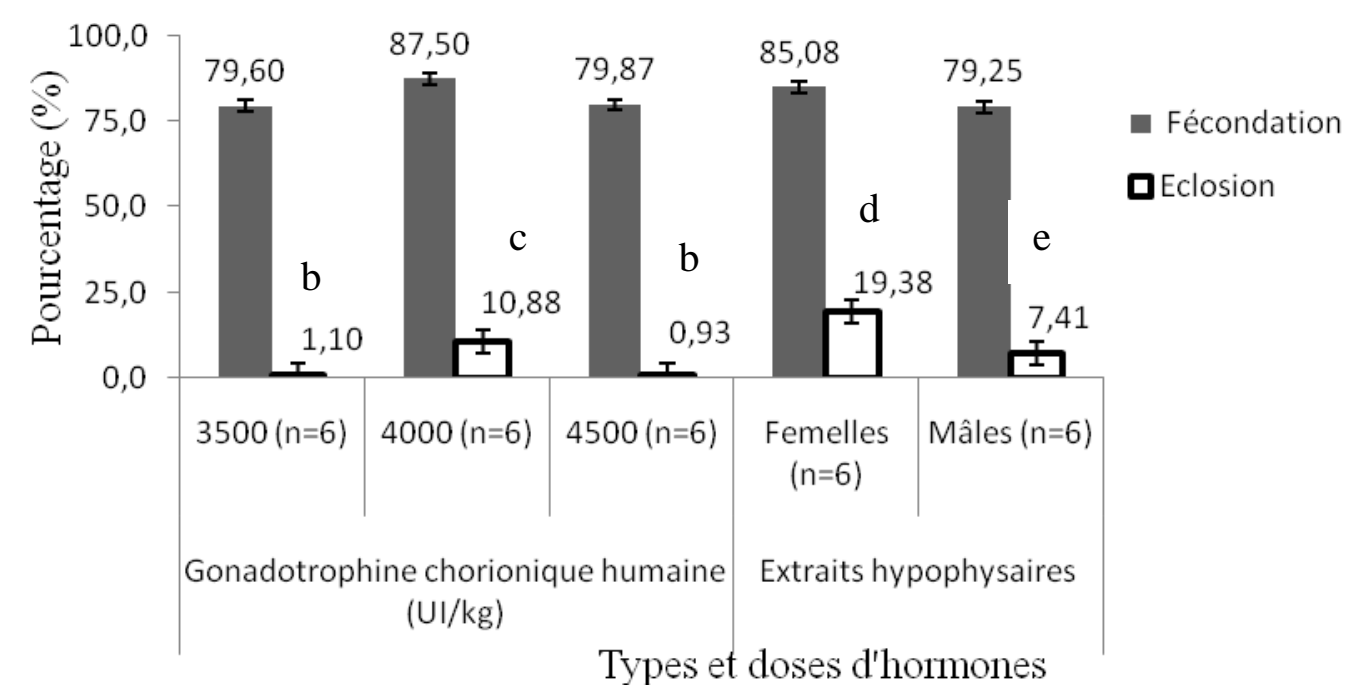

Figure 4: Taux de fécondation et d'éclosion en fonction du type et dose d'hormones. a, b, c, d, e : les histogrammes affectés des mêmes lettres ne sont pas significativement différentes $(\mathrm{p}>0,05) ; \mathrm{n}=$ nombre d'observations; $\mathrm{I}=$ écart type.

\section{DISCUSSION}

Temps de latence et d'incubation

Le temps de latence a diminué avec la température et plus long comparativement à d'autres espèces déjà étudiées. En effet, pour une température de l'eau de $25^{\circ} \mathrm{C}$, il a été en moyenne de 22 heures contre 18 heures et 11 heures respectivement chez Heterobranchus bidorsalis (Alla et al., 2010) et Clarias gariepinus (Ahotondji, 2012), le temps de latence également pour une température moyenne de l'eau de $21^{\circ} \mathrm{C}$ a été de 30 heures contre 18 heures chez Clarias gariepinus (Ahotondji, 2012). Le temps d'incubation a diminué avec la température, les valeurs obtenues restent supérieures à 23 heures à 27 ${ }^{\circ} \mathrm{C}$ et à 46 heures à $21{ }^{\circ} \mathrm{C}$ rapportées par Ahotondji en 2012 chez Clarias gariepinus.

\section{Diamètre et poids des ovocytes}

Au terme de l'essai, il est apparu que l'hormone gonadotrophine chorionique humaine aux doses de 3500, 4000 et 4500
UI/kg et les extraits hypophysaires mâles et femelles ont affecté le poids des ovocytes. Une des explications serait la quantité d'hormone reçue par les femelles et plus spécifiquement leur âge différent. Par ailleurs, les valeurs de diamètre des ovocytes $(1.65 \pm$ 0.07 à $1.73 \pm 0.15 \mathrm{~mm}$ ) ont été comparables à $1.56 \pm 0.13$ à $2.5 \pm 0.17 \mathrm{~mm}$ obtenues par Zango et al. (2015) chez la même espèce prête à pondre et provenant des cours d'eau Menoua et Nkam dans la région de l'Ouest-Cameroun, elles ont été néanmoins supérieures à $0.5 \pm$ $0.01-1.6 \pm 0.06 \mathrm{~mm}$ chez Clarias gariepinus capturé dans les mêmes conditions d'une part et supérieures également à $1.39 \pm 0.14-1.50$ $\pm 0.07 \mathrm{~mm}$ chez Heterobranchus bidorsalis en conditions d'élevage rapportées par Alla et al. (2010).

Fécondités moyennes absolue et relative à différents types et doses d'hormone

Les fécondités absolue et relative des femelles induites au hCG à 4000 UI/g de 
poids vif $(8326 \pm 4651$ ovocytes et $38 \pm 15$ ovocytes / g O) ne diffèrent pas de celles des femelles traitées aux extraits hypophysaires. Ce résultat corrobore avec celui de Rukera et al. (2005) chez Clarias gariepinus (105541 \pm 3644 ovocytes chez les femelles induites à l'ovaprim contre $99897 \pm 7551$ des femelles ayant reçu des extraits hypophysaires). Cependant, elles ont été inférieures à $43989 \pm$ 19766 ovocytes et $168 \pm 38$ ovocytes / g o chez Clarias gariepinus (Chikou et al., 2006) en milieu naturel, également inférieures à $140440 \pm 82168$ ovocytes et $47.30 \pm 17.94$ ovocytes / g 9 chez Heterobranchus bidorsalis (Alla et al., 2010) et à $99897 \pm$ 7551 ovocytes chez Clarias gariepinus (Rukera et al., 2005) en conditions d'élevage. Ces différences traduisent chez les espèces les stratégies de reproduction différentes. En effet les fécondités élevées trouvées en milieu naturel s'expliquent par le fait que les poissons - chats développent une stratégie pour se maintenir sous une forte pression de pêche en produisant beaucoup d'œufs.

\section{Taux de fécondation et d'éclosion}

Le taux de fécondation obtenu avec la dose de $4000 \mathrm{UI} / \mathrm{kg}$ de l'hormone hCG a été de $2,42 \%$ supérieur à celui trouvé avec l'extrait hypophysaire d'origine femelle. Ce résultat pourrait se justifier par la différence de concentration en hormones hypophysaires agissant dans la maturation ovocytaire finale entre les géniteurs. Cependant, il a été de 21, $83 \%$ supérieur à celui rapporté par Rukera (2004) chez Clarias gariepinus. Il a également été supérieur à $75.97 \%$ rapportés par Tiogue et al. (2008). Ces différences seraient liées à l'espèce. En considérant le taux d'éclosion, les résultats enregistrés avec la gonadotrophine chorionique humaine ont montré que la dose optimale se situerait entre 3500 et de $4500 \mathrm{UI} / \mathrm{kg}$. Cette observation est proche de celle de Rukera (2004) chez qui la dose optimale a été de $4000 \mathrm{UI} / \mathrm{kg}$ pour Clarias gariepinus. Par ailleurs, les doses plus élevées ont donné des résultats inférieurs à celui de la dose optimale. Cette différence pourrait s'expliquer par le phénomène de «feed back». Pour l'extrait hypophysaire, le taux d'éclosion obtenu avec l'extrait hypophysaire d'origine femelle a été de 11 , $97 \%$ élevé à celui provenant des mâles. A Ces différences observées peuvent être attribuées au sexe. En effet, la maturation finale des ovocytes serait plus complète avec l'utilisation de l'extrait hypophysaire prélevé chez les femelles. Les taux d'éclosion obtenus ont été différents de ceux rapportés par Cacot (2006) qui n'avait enregistré aucune éclosion chez Clarias jaensis. En effet, l'état physiologique des géniteurs et la température auraient affecté le taux d'éclosion. De plus, le taux d'éclosion obtenu à la fin de l'essai a été de 24,62\% faible à celui enregistré par Rukera et al. (2005) chez Clarias gariepinus. La différence entre les espèces pourrait justifier ces résultats.

\section{Conclusion}

Le poids des ovules, les taux de fécondation et d'éclosion ont été affecté significativement par le type et la dose d'hormones. Ainsi, ils ont été significativement élevés avec l'extrait hypophysaire provenant des femelles. Le taux de fécondation a été comparable entre les types et doses d'hormone.

\section{CONFLIT D'INTERETS}

Les auteurs déclarent qu'il n'y a aucun conflit d'intérêt.

\section{CONTRIBUTIONS DES AUTEURS}

Tous les auteurs ont participé au choix de l'espèce, à la conception du sujet, à la collecte des données sur le terrain et à la rédaction du manuscrit.

\section{REMERCIEMENTS}

Nous exprimons toute notre gratitude à Monsieur G. Olivier, pêcheur à Lelem pour 
avoir fourni les géniteurs, MM. Bernard YOUDOM et Michel DIOGNE que nous remercions vivement pour leur entière disponibilité et la mise à notre disposition des infrastructures d'acclimatation et de reproduction des poissons.

\section{REFERENCES}

Ahotondji A. 2012. Renforcement des capacités nationales des petits producteurs dans la production intensive d'alevins de clarias. Rapport Technique Définitif. Projet ACP FISH II, Activité 4.1, Projet $\mathrm{N}^{\circ}$ : A8bDOI: acpfish2-eu.org/.../RTF\%20Projet\% 20A8\%20ACP\%20FISH\%20II\%20G

Alla YL, Ouattara M, Blé MC, Atse BC. 2010. Etude de la fécondité du silure Africain heterobranchus bidorsalis (Geoffroy Saint - Hilaire, 1840) en conditions d'élevage. Tropicultura, 28: (1): 31-36 www. tropicultura.org/text/v28n1/31.pdf

Baras E, Jobling M. 2002. Dynamics of intracohort cannibalism in the culture fish. Aquaculture Research, 33: 461479. DOI: https://orbi.ulg.ac.be/ bitstream/.../Baras\%20et\%20al.p

Cacot P. 2006. Contribution à l'amélioration de la production d'alevins au Cameroun: Essais de reproductions et d'élevage de nurserie avec Clarias gariepinus et deux autres espèces. Mission effectuée du 7 au 29 novembre 2006 dans le cadre du projet ATPCIROP animé par Olivier MIKOLASEK en collaboration avec la station de l'IRAD dirigée par David NGUENGA, Foumban, Cameroun. 60p. lists.stir.ac.uk/pipermail/sarnissafrench-aquaculture/.../attachment.pdf

Chikou A. 2006. Etude de la démographie et de l'exploitation halieutique de six espèces de poissons chat (téléostéens, Siluriformes) du Delta de l'Ouemé au Bénin, Thèse de Doctorat, 435p. https://pure.fundp.ac.be/ws/files/44872 53/Studentthesis-2007.pdf

Dadebo E, Ahlgren G, Ahlgren I. 2003. Aspects of reproductive biology of Labeo horie Heckel (Pisces: Cyprinidae) in Lake Chamo, Ethiopia. Afr. J. Ecol., 41: 31-38.

FAO. 2012. Situation mondiale des pêches et l'aquaculture. FAO, Rome, 28p. www.fao.org/docrep/016/i2727f/i2727f .pdf

FAO. 2014. La situation mondiale des pêches et de l'aquaculture. FAO, Rome, Italie, 2p. www.fao.org/2/i3720f \#SOFIA2014

Fostier A, jalabert B. 2004. Domestication et reproduction chez les poissons. INRA Productions Animales, 17(3): 199204. https://www6.inra.fr/ productionsanimales/.../Prod_Anim_2004_17_3_0

Lazard J, Cacot P, Slembrouck J, Legendre M. 2009. La culture des Pangasiidae. Cahiers Agricultures, 18: 164 - 173. www.documentation.ird.fr/hor/fdi:0100 46191

Nguenga D. 2000. Partial gonadectomy in the catfish Heterobranchus longifilis (Teleostei, Clariidae): Regeneration time, quality and quantily of postsurgical sperm production. The Israeli Journal of AquacultureBamidgeh, 4(52): 167-172. DOI: https://www.researchgate.net/.../289805 070_Partial_g

Philippart J-C. 1975. Dynamique des populations de poissons non exploités. In Dynamique des Populations de Vertébrés, Lamotte $\mathrm{M}$, Bourlière $\mathrm{F}$ (eds). Ed. Masson : Paris ; 292-394.

Rukera TS, Micha JC, Ducarne C. 2005. Essais d'adaptation de production massive de juvénilesde Clarias gariepinus en conditions rurales. Tropicultura, 23(4) : 231-244.

Sorgeloos P, 2014. L'aquaculture a-t-elle le potentiel pour devenir la « biotechnologie bleue » dans l'avenir ? 
Cah. Agric., 23: 53-64. DOI: 10.1684/agr.2014.0683

Tiogue CT, Nguenga D, Tomedi MTE, Tchoumboue J. 2008. Quelques performances reproductives et taux de survie de deux souches du poisson chat africain Clarias gariepinus (Burchell, 1822) et de leurs croisés à Koupa - Matapit. Int. J. Biol. Chem. $\begin{array}{lllll}\text { Sci. 2(4): } 469 \quad-\quad 477 \text {; } & 4\end{array}$ http://indexmedicus.afro.who.int

Yalçin Ş, Solak K, Akyurt I. 2001. Certain reproductive characteristics of the catfish (Clarias gariepinus Burchell,
1822) living in the River Asi, Turky. Turk J. Zool., 25: 453-460.

Zango p, Tomedi TEM, Oben ML, Pouomogne V, Nguenga D, Tchoumboue J, Efole ET, Mikolasek O. 2015. Comparing Reproductive Characteristics of two Catfish Species Clarias gariepinus and Clarias jaensis of The Western region of Cameroon. Journal of Multidisciplinary Engineering Science and Technology (JMEST) 2(12): December - 2015. DOI: www.jmst.org. 